\title{
Common Femoral Vein
}

National Cancer Institute

\section{Source}

National Cancer Institute. Common Femoral Vein. NCI Thesaurus. Code C154771.

A vein that accompanies the common femoral artery and is formed by the confluence of the femoral vein and the deep femoral vein. 